\title{
Integrated imaging of adrenal oncocytoma: a case of diagnostic challenge
}

\author{
Milena Coppola ${ }^{1}$, Valeria Romeo ${ }^{1}$, Francesco Verde ${ }^{1}$, Giorgio Raia ${ }^{1}$, Ciro Gabriele Mainolfi ${ }^{1}$, Giovanni Aprea ${ }^{2}$, \\ Elia Guadagno ${ }^{1}$, Carlo Cavaliere ${ }^{3}$, Dario Baldi ${ }^{3}$, Andrea Soricelli ${ }^{3}$, Pier Paolo Mainenti ${ }^{4}$, Simone Maurea ${ }^{1}$ \\ ${ }^{1}$ Department of Advanced Biomedical Sciences, ${ }^{2}$ Department of General and Mini-invasive Surgery, University of Naples "Federico II", Naples, \\ Italy; ${ }^{3}$ IRCCS SDN, Naples, Italy; ${ }^{4}$ Institute of Biostructures and Bioimaging of the National Research Council (CNR), Naples, Italy
}

Correspondence to: Valeria Romeo, MD, PhD. Department of Advanced Biomedical Sciences, University of Naples Federico II, via S. Pansini 5, 80131, Naples, Italy. Email: valeria.romeo@unina.it.

Submitted Apr 11, 2019. Accepted for publication Jun 26, 2019.

doi: 10.21037/qims.2019.06.20

View this article at: http://dx.doi.org/10.21037/qims.2019.06.20

\section{Introduction}

Oncocytomas are in most cases benign tumors, usually arising in kidneys as well as thyroid, parathyroid, salivary or pituitary glands $(1,2)$. This kind of tumor is extremely rare in adrenals and it is usually discovered as incidental finding because is nonfunctional; however, in about $20 \%$ of cases features suggestive for malignancy and/or adrenal hypersecretion have been reported (3). Tumor size criterion often allows to differentiate a benign from a malignant adrenal mass, but in oncocytic neoplasm neither size lesion nor imaging findings are useful for this purpose; indeed, usually oncocytomas present as large adrenal masses and surgical excision is often required $(4,5)$. Therefore, a careful diagnostic evaluation is recommended for lesion characterization as well as for the assessment of potential malignancy.

We aimed to describe imaging features of adrenal oncocytoma and to highlight the diagnostic pitfalls that can be encountered in the interpretation of images obtained from different modalities reporting a case of a female patient evaluated in our institution.

\section{Case report}

A 34-year-old-woman suffering from recurrent cystitis underwent an abdominopelvic ultrasound. As incidental finding, a lobulated solid adrenal mass $(47 \mathrm{~mm} \times 30 \mathrm{~mm})$ was detected. The patient underwent laboratory evaluation of adrenal function and no abnormal cortical and/ or medullary hormonal values were detected (Table 1). DECT (tube voltages of 150 and $100 \mathrm{kVp}$ ) acquired before and after iodinate contrast medium administration was performed showing a solid nodular patchy mass of the right adrenal gland $(39 \mathrm{~mm} \times 24 \mathrm{~mm} \times 40 \mathrm{~mm})$ with inner septa, pre-contrast attenuation coefficients suggestive of solid tissue $(40 \mathrm{HU})$ and a fat fraction of $15.5 \%$. The lesion had an irregular vascularization due to the presence of hemorrhagic foci, showed a rapid outflow of contrast medium after 10 minutes with an Absolute Wash-out rate $\left(\mathrm{AWO}_{10}\right)$ of $43 \%$, and a Iodine density of $3.0 \mathrm{mg} / \mathrm{mL}$ (57\%). Moreover, a decrease of attenuation coefficients was observed between low $(115 \mathrm{HU})$ and high $(67 \mathrm{HU})$ energies in the portal phase. The mass was not separated from the liver parenchyma, inferior cava vein and superior pole of right kidney (Figure 1). To further characterize the adrenal lesion, a contrast-enhanced magnetic resonance imaging (MRI) examination was performed, showing: (I) absence of intracellular fat on in \& out-of-phase images; (II) hypointense hemorrhagic components on T2weighted images; (III) significant restriction on DWI; (IV) and increased contrast enhancement on dynamic sequences with an $\mathrm{AWO}_{10}$ rate $37.7 \%$ (Figures 2,3). Finally, a 2-(18F)FDG PET/CT scan was performed to assess the metabolic activity of the lesion, showing high (SUVmax $=26.4$ ) tracer uptake within the nodule (Figure 4). On the basis of DECT, 2-( $\left.{ }^{18} \mathrm{~F}\right)$ FDG PET/CT and MRI features a malignant nature of the lesion was suggested. The patient underwent surgical excision of the right adrenal nodule; 
however, histology demonstrated a benign cortical adrenal oncocytoma (Figure 5).

\section{Discussion}

We report a case of an adrenal oncocytoma in which a significant increase of $2-\left({ }^{18} \mathrm{~F}\right) \mathrm{FDG}$ uptake was observed. DECT, MRI and 2-( $\left.{ }^{18} \mathrm{~F}\right) \mathrm{FDG}$ PET/CT features were also suggestive for malignancy, but histology after surgical excision demonstrated a benign nature.

Adrenal oncocytoma is a very rare disease, observed

Table 1 Laboratory results of adrenal function

\begin{tabular}{lcc}
\hline Laboratory test & Patient's value & Normal range \\
\hline Cortical function & & \\
Cortisol (hours 8$)(\mathrm{ng} / \mathrm{mL})$ & 100 & $50-200$ \\
Urinary cortisol $(\mu \mathrm{g} / 24 \mathrm{~h})$ & 17 & $10-130$ \\
ACTH $(\mathrm{pg} / \mathrm{mL})$ & 416 & $35-430$ \\
DHEA-S $(\mu \mathrm{g} / \mathrm{dL})$ & 49 & $40-432$ \\
Aldosterone $(\mathrm{pg} / \mathrm{mL})$ & & \\
Medullary function & 2.1 & $<27.0$ \\
Urinary adrenaline $(\mu \mathrm{g} / 24 \mathrm{~h})$ & 56.1 & $<97.0$ \\
Urinary noradrenaline $(\mu \mathrm{g} / 24 \mathrm{~h})$ & 83 & $<320$ \\
Urinary metanephrine $(\mu \mathrm{g} / 24 \mathrm{~h})$ & 21.0 & $20-190$ \\
Plasmatic adrenaline $(\mathrm{pg} / \mathrm{mL})$ & 133.0 & $70-480$ \\
Plasmatic noradrenaline $(\mathrm{pg} / \mathrm{mL})$ & & \\
\hline
\end{tabular}

within the adrenal cortex and frequently encountered in female patients. Up to $70 \%$ of oncocytomas are nonfunctional and the majority is incidentally detected (2). In a minority of cases these tumors may be symptomatic due to adrenal hypersecretion. The histological characteristic of oncocytoma is the predominance of oncocytes, that are epithelial cells characterized by abundant granular eosinophilic cytoplasm packed with plentiful mitochondria. Some tumors display areas of hemorrhage and necrosis, while the classic central radiating scar that has been described in most renal oncocytomas is not always present in adrenocortical oncocytoma (3).

Imaging characterization of adrenal tumors is based on CT and MRI criteria consisting of tumor size, morphological features, pre- and post-contrast CT densitometry as well as MRI pre-and post-contrast signal intensity characteristics (4). When CT and/or MRI images are uncertain for tumor characterization, functional radionuclide modalities may be used with $2-\left({ }^{18} \mathrm{~F}\right) \mathrm{FDG}$ PET/CT or PET-MRI hybrid imaging (6). According to the current literature in which tumor size is considered less sensitive than imaging features in predicting malignancy, in our case a significantly high maximum diameter $(4 \mathrm{~cm})$ was observed in a benign lesion (5). CT and MRI features were also suggestive for malignancy. Indeed, pre-contrast CT densitometry was $40 \mathrm{HU}$ and post-contrast CT scan demonstrated a solid lesion with an indeterminate wash-out rate of $43 \%$, inferior to thresholds commonly reported in the literature for the diagnosis of adenoma, the most common benign adrenal lesions (7). Furthermore, the lesion showed a significant decrease
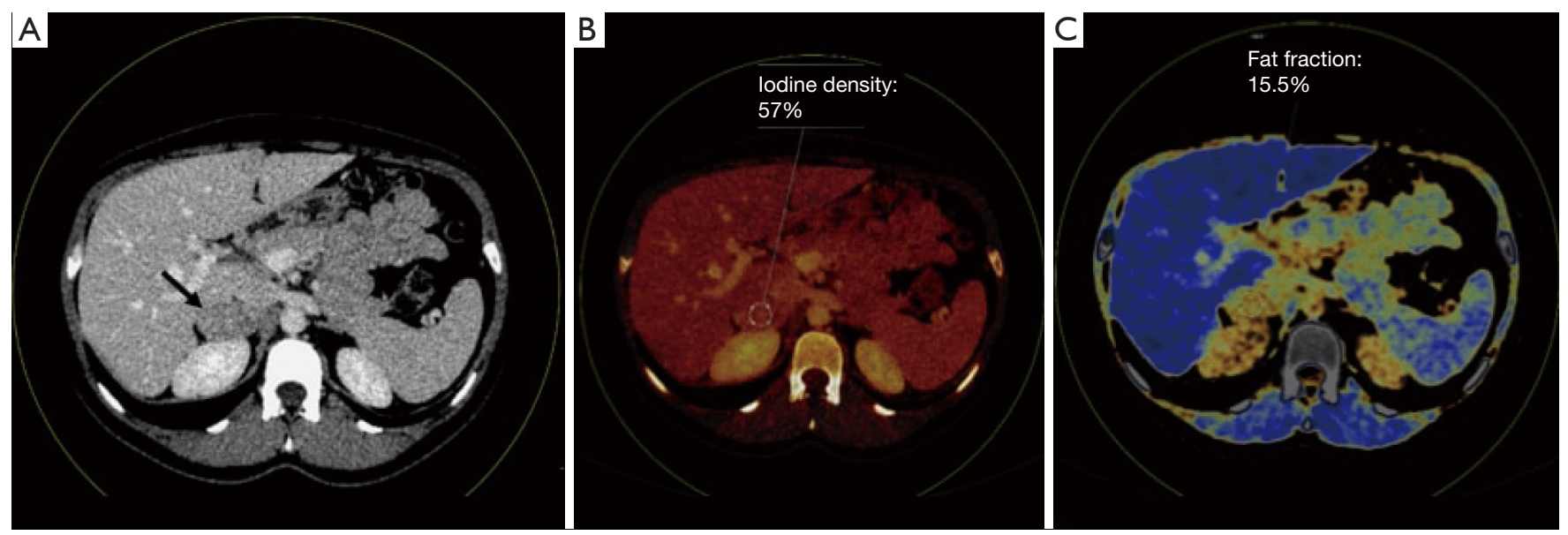

Figure 1 DECT; axial images on the portal phase (A); iodine map (B); fat map (C). The right adrenal lesion (black arrow) showed significant contrast-enhancement with an iodine density of $57 \%$ and a fat fraction of $15.5 \%$. DECT, dual energy computed tomography. 

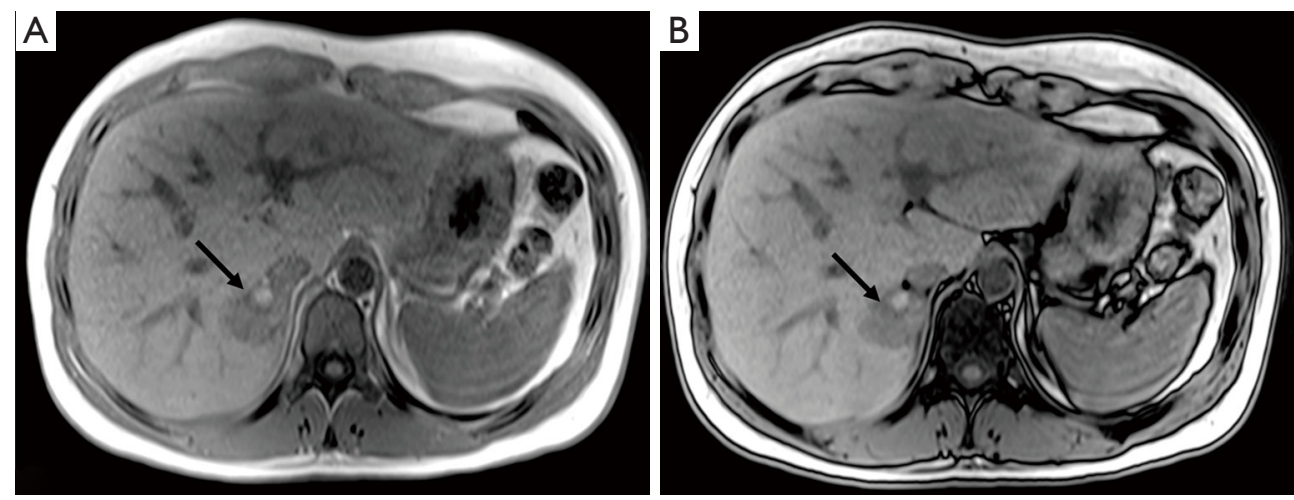

Figure 2 T1-weighted chemical-shift (CS) MRI; CS in-phase (A) and CS out-of-phase (B): a round, right adrenal mass without signal intensity loss is showed (black arrows). MRI, magnetic resonance imaging.
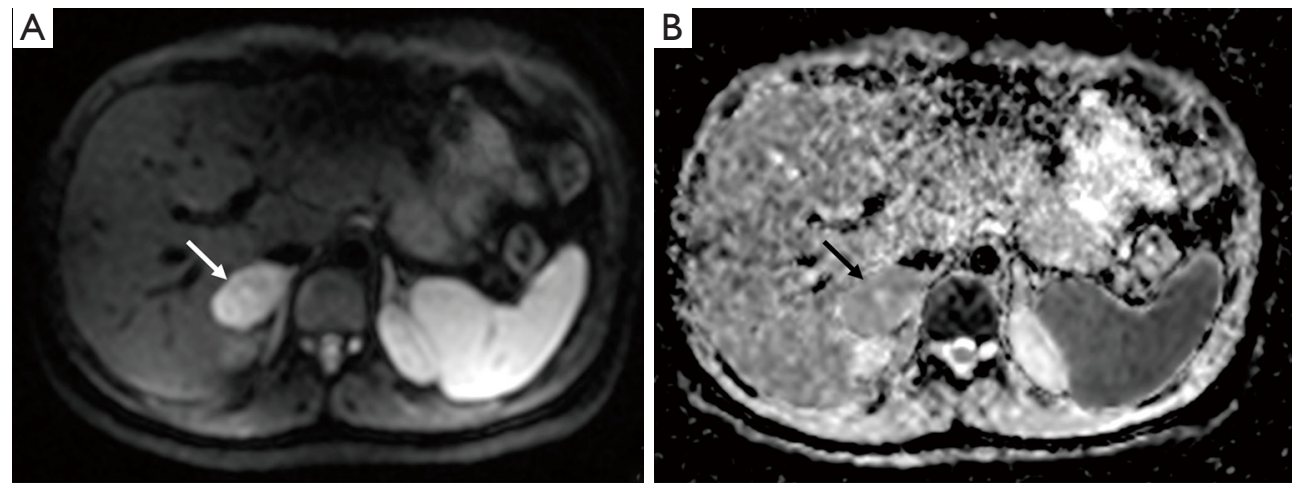

Figure 3 Diffusion-weighted imaging (DWI) MRI; DWI (A) and apparent diffusion coefficient (ADC) map (B): a significant restricted diffusivity of the adrenal mass (white arrow in A and black arrow in $\mathrm{B}$ ) is detected (ADC value $=1.1$ ). MRI, magnetic resonance imaging.

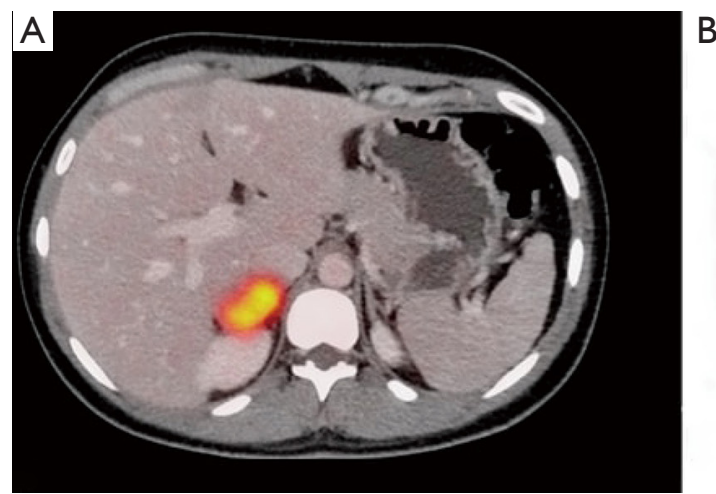

\section{B}

Figure $42-\left({ }^{18} \mathrm{~F}\right)$ FDG PET/CT; fused PET/CT image (A) shows a round right adrenal mass with significantly increased FDG uptake (SUV $\max =26)(B)$. PET/CT, positron emission tomography/computed tomography. 

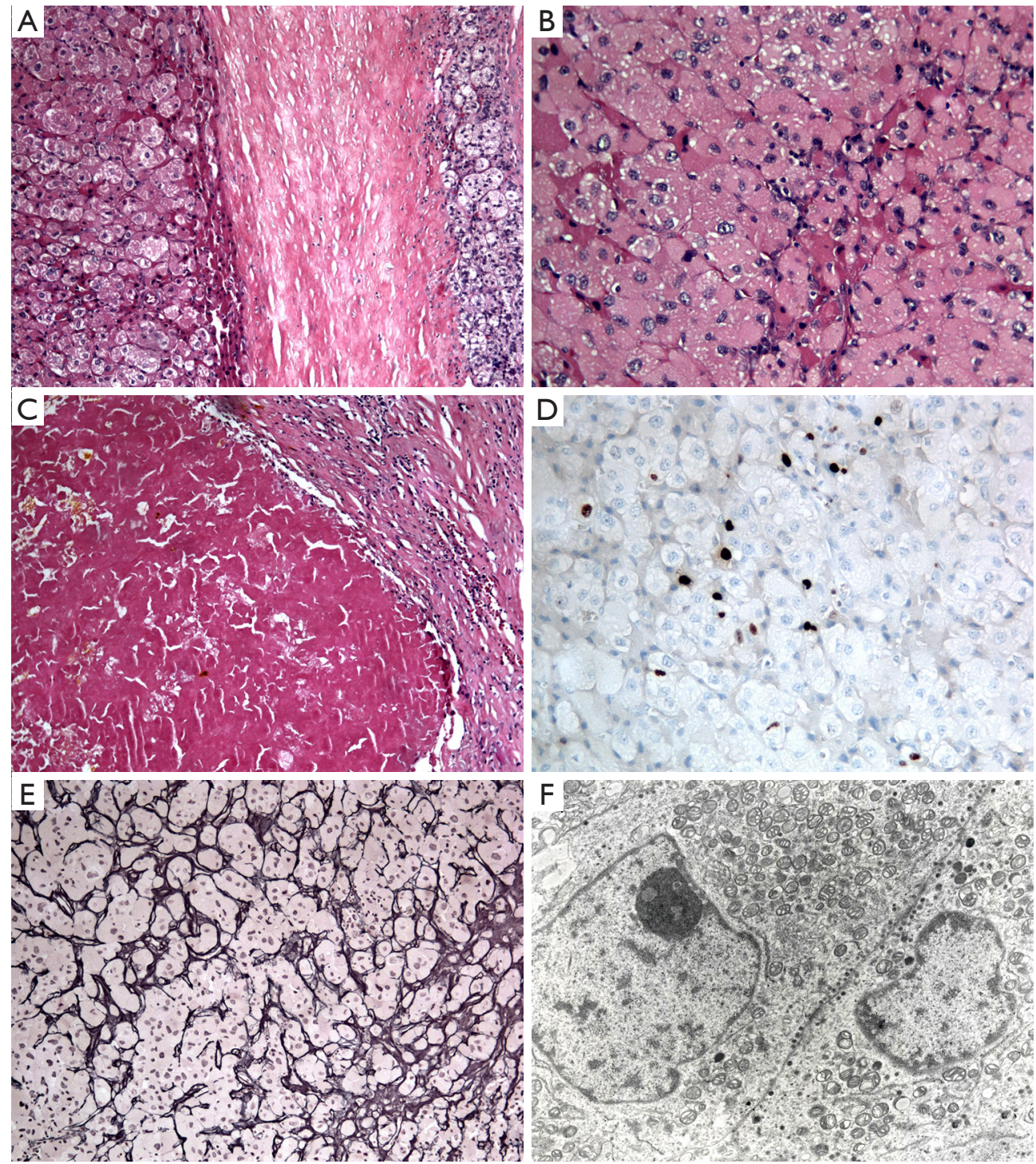

Figure 5 Hematoxylin \& eosin stain: (A) 10×, (B,C) 40× magnification. Ki67 immunohistochemistry: (D) 40× magnification; Gordon Sweet Reticulin stain: (E) 20x magnification. Ultrastructure: (F) 4,400 magnification. A thick capsule (A) clearly delimited the normal adrenal gland (on the right) from a neoplastic proliferation composed by large cells with dense eosinophilic cytoplasm (B) and with occasional nuclear pleomorphism and prominent nucleoli; degenerative changes following haemorrhage were evident in one field (C); Ki67 cellular proliferative index was calculated around 4\% in a "hotspot" area (D); reticulin histochemical staining revealed an intact network (E) as it is usually observed in adrenal cortical adenoma; ultrastructural examination showed that the cytoplasm of neoplastic cells was full of mitochondria (F).

of attenuation coefficients at $150 \mathrm{kVp}$ as compared to $100 \mathrm{kVp}$, a fat fraction of $15.5 \%$ and an iodine density of $3.0 \mathrm{mg} / \mathrm{mL}$, features that have been previously observed in adrenal metastases (8). In this setting, in a previous study conducted by Martin and colleagues on adrenal adenomas and metastases using DECT, adrenal adenomas demonstrated a significantly higher fat fraction $(34.2 \%)$ and lower iodine density as compared to metastases $(34.2 \%$ vs. $10.7 \%$ and 1.35 vs. $3.2 \mathrm{mg} / \mathrm{mL}$ respectively) (9). As also previously suggested, when CT and MRI findings fail to differentiate an adrenal oncocytoma from adrenal malignant one, $2-\left({ }^{18} \mathrm{~F}\right) \mathrm{FDG}$ PET/CT may be helpful to differentiate benign from malignant non-functioning adrenal masses. However, 
this imaging technique could be misleading in cases of adrenal oncocytomas since increased $2-\left({ }^{18} \mathrm{~F}\right) \mathrm{FDG}$ uptake may occur (6-16). These peculiar findings of adrenal oncocytoma could be probably explained by the different cellularity (related to the absence of intracellular fat), and the high metabolic activity (probably underpinning a greater blood supply) of oncocytomas as compared to adenomas, similar to those of malignant lesions. Similarly, MRI features consisted of absence of intracellular lipid, presence of hemorrhagic foci, significant restriction on DWI and post-contrast dynamic characteristics with indeterminate wash-out rate (10).

The adrenal oncocytoma we described in our case showed similar characteristics as compared to those previously reported in literature in last ten years such as higher frequency in female, fortuitous findings, large lesion size and high 2-( $\left({ }^{18} \mathrm{~F}\right) \mathrm{FDG}$ uptake (13-15). A false positive finding of metaiodobenzylguanidine scintigraphy has been also described in such lesions (17). In addition to previous studies, we also reported DECT imaging features of adrenal oncocytomas in terms of CT densitometry and fat fraction that also failed to properly characterize the lesion.

Since the imaging characterization of adrenal oncocytoma effectively represents a diagnostic challenge, the definitive diagnosis of adrenal oncocytoma could be pre-operatively secured by fine-needle aspiration cytology and/or biopsy (18). Adrenalectomy is the treatment of choice, but the prognosis depends on immunohistochemical classification system that classifies adrenal oncocytoma on the basis of the risk of recurrence, metastasis, and death $(2,7,19)$.

In conclusion, imaging findings alone, even with the addition of more advanced imaging techniques, are not able to characterize adrenal oncocytoma. Morpho-functional integrated imaging is recommended to characterize adrenal tumors, but since $2-\left({ }^{18} \mathrm{~F}\right) \mathrm{FDG}$ uptake may often occur in adrenal oncocytoma, malignancy may be overestimated. Therefore, this peculiar finding should be taken into account for appropriate imaging interpretation of incidental adrenal lesions, especially when encountered in young females.

\section{Acknowledgments}

None.

\section{Footnote}

Conflicts of Interest: The authors have no conflicts of interest to declare.

\section{References}

1. Mearini L, Del Sordo R, Costantini E, Nunzi E, Porena M. Adrenal oncocytic neoplasm: a systematic review. Urol Int 2013;91:125-33.

2. Wong DD, Spagnolo DV, Bisceglia M, Havlat M, McCallum D, Platten MA. Oncocytic adrenocortical neoplasms-a clinico- pathologic study of 13 new cases emphasizing the importance of their recognition. Hum Pathol 2011;42:489-99.

3. Chang A, Harawi SJ. Oncocytes, oncocytosis, and oncocytic tumors. Pathol Annu 1992;27:263-304.

4. Kandathil A, Wong KK, Wale DJ, Zatelli MC, Maffione AM, Gross MD, Rubello D. Metabolic and anatomic characteristics of benign and malignant adrenal masses on positron emission tomography/computed tomography: a review of literature. Endocrine 2015;49:6-26.

5. Yoo JY, McCoy KL, Carty SE, Stang MT, Armstrong MJ, Howell GM, Bartlett DL, Tublin ME, Yip L. Adrenal Imaging Features Predict Malignancy Better than Tumor Size. Ann Surg Oncol 2015;22 Suppl 3:S721-7.

6. Riola-Parada C, García-Cañamaque L, Pérez-Dueñas V, Garcerant-Tafur M, Carreras-Delgado JL. Simultaneous PET/MRI vs PET/CT in oncology. A systematic review. Rev Esp Med Nucl Imagen Mol 2016;35:306-12.

7. Angelelli G, Mancini ME, Moschetta M, Pedote P, Pignataro P, Scardapane A. MDCT in the differentiation of adrenal masses: comparison between different scan delays for the evaluation of intralesional washout. ScientificWorldJournal 2013;2013:957680.

8. Gupta RT, Ho LM, Marin D, Boll DT, Barnhart HX, Nelson RC. Dual-energy CT for characterization of adrenal nodules: initial experience. AJR Am J Roentgenol 2010;194:1479-83.

9. Martin SS, Weidinger S, Czwikla R, Kaltenbach B, Albrecht MH, Lenga L, Vogl TJ, Wichmann JL. Iodine and Fat Quantification for Differentiation of Adrenal Gland Adenomas From Metastases Using ThirdGeneration Dual-Source Dual-Energy Computed Tomography. Invest Radiol 2018;53:173-8.

10. Romeo V, Maurea S, Guarino S, Mainenti PP, Liuzzi R, Petretta M, Cozzolino I, Klain M, Brunetti A. The role of dynamic post-contrast T1-w MRI sequence to characterize lipid-rich and lipid-poor adrenal adenomas in comparison to non-adenoma lesions: preliminary results. Abdom Radiol (NY) 2018;43:2119-29.

11. Podetta M, Pusztaszeri M, Toso C, Procopiou M, Triponez F, Sadowski SM. Oncocytic Adrenocortical Neoplasm with 
Concomitant Papillary Thyroid Cancer. Front Endocrinol (Lausanne) 2018;8:384.

12. Shenouda M, Brown LG, Denning KL, Pacioles T. A Case of Oncocytic Adrenocortical Neoplasm of Borderline (Uncertain) Malignant Potential. Cureus 2016;8:e638.

13. Kakita-Kobayashi M, Usui T, Sasano H, Shimatsu A. 18F-FDG-PET-positive adrenal tumour. BMJ Case Rep 2015;2015. doi: 10.1136/bcr-2015-209379.

14. Son SH, Lee SW, Song BI, Jang YJ, Park JY, Jeong SY, Ahn BC, Lee J. Recurrence of a functional adrenocortical oncocytoma of borderline malignant potential showing high FDG uptake on 18F-FDG PET/CT. Ann Nucl Med 2014;28:69-73.

15. Sato N, Nakamura Y, Takanami K, Ono Y, Omata K, Morimoto R, Satoh F, Ise K, Yamada S, Kasajima A, Fujishima F, Watanabe M, Arai Y, Sasano H. Case report: adrenal oncocytoma associated with markedly increased

Cite this article as: Coppola $M$, Romeo V, Verde F, Raia G, Mainolfi CG, Aprea G, Guadagno E, Cavaliere C, Baldi D, Soricelli A, Mainenti PP, Maurea S. Integrated imaging of adrenal oncocytoma: a case of diagnostic challenge. Quant Imaging Med Surg 2019;9(11):1896-1901. doi: 10.21037/ qims.2019.06.20
FDG uptake and immunohistochemically positive for GLUT1. Endocr Pathol 2014;25:410-5.

16. Fassnacht $M$, Kenn W, Allolio B. Adrenal tumors: how to establish malignancy? J Endocrinol Invest 2004;27:387-99.

17. Padberg BC, Rordorf T, Suter SL, Pfeiffer D, Wild D, Schröder S. 123I-Metaiodobenzylguanidine (MIBG) scintigraphy: paradoxical positivity in an oncocytic adrenocortical carcinoma. Pathologe 2007;28:281-4.

18. Krishnamurthy S, Ordóñez NG, Shelton TO, Ayala AG, Sneige N. Fine-needle aspiration cytology of a case of oncocytic adrenocortical carcinoma. Diagn Cytopathol 2000;22:299-303.

19. Bisceglia M, Ludovico O, Di Mattia A, Ben-Dor D, Sandbank J, Pasquinelli G, Lau SK, Weiss LM. Adrenocortical oncocytic tumors: report of 10 cases and review of the literature. Int J Surg Pathol 2004;12:231-43. 